\title{
Adaptive temperature controller for plastic extrusion process
}

\section{Control de temperatura adaptable para proceso de extrusión de plástico}

\author{
GUERRERO, Jesús†'*', GONZÁLEZ, Julio“' and CHIMAL, Martin’ \\ 'Universidad Politécnica de Juventino Rosas, Automotive Systems Engineering Department, Mexico.
}

"Universidad Politécnica de Juventino Rosas, Plastics Engineering Department, Mexico.

ID $1^{\text {st }}$ Author: Jesús, Guerrero / ORC ID: 0000-0002-5873-8986, CVU CONACYT-ID: 490978

ID $1^{\text {st }}$ Coauthor: Julio, González

ID $2^{\text {nd }}$ Coauthor: Martin, Chimal

DOI: $10.35429 /$ JTP.2020.18.6.10.17

Received July 30, 2020; Accepted December 17, 2020

\begin{abstract}
In this paper, an adaptive temperature controller for the plastic extrusion process is designed. The proposed controller aims to solve the set-point regulation problem and the temperature trajectory tracking of a plastic extrusion process. The controller is an adaptive version of the First Sliding Mode Control which is robust towards parametric uncertainties and external disturbances. Also, the finite time convergence is demonstrated by Lyapunov arguments. Finally, the effectiveness of the proposed controller under several scenarios is demonstrated by computer simulations.
\end{abstract}

Adaptive controller, Sliding modes control, Extrusion process, Temperature control

\begin{abstract}
Resumen
En este artículo, se diseña un control adaptable para el proceso de extrusión de plástico. El controlador propuesto está enfocado en resolver el problema de regulación, así como también el seguimiento de perfiles de temperature en el proceso de extrusion de plástico. El controlador es una versión adaptable de control por modos deslizantes de primer orden, el cual es robusto ante perturbaciones paramétricas y externas. También, se demuestra la estabilidad del controlador propuesto mediante la teoría de Lyapunov. Finalmente, se demuestra la efectividad del controlador por medio de diferentes escenarios en simulación por computadora.
\end{abstract}

Control adaptable, Control por modos deslizantes, Proceso de extrusión, Control de temperatura

Citation: GUERRERO, Jesús, GONZÁLEZ, Julio and CHIMAL, Martin. Adaptive temperature controller for plastic extrusion process. Journal of Technological Prototypes. 2020. 6-18:10-17.

\footnotetext{
* Correspondence to Author (Email: jguerrerot_pa@upjr.edu.mx)

$\dagger$ Researcher contributing first author
} 


\section{Introduction}

The extrusion process refers to any transformation operation in which molten material is forced through a nozzle to produce an article with a constant cross-section and definite length. In addition to plastics, many other materials are processed by extrusion, such as metal, ceramics or food, obtaining very varied products such as aluminium window frames, pipes, food, and so on (the reader can be referred to (Hammond (2020), Guzmán (2020), Guerrero et. al. (2020), Urra (2020), and Marquina (2020)). From the point of view of plastics engineering, extrusion is one of the most important transformations processes. The plastic extrusion process is carried out in machines called extruders. Although there are extruders of various types, the most commonly used are the single screw.

In the extrusion process, the polymer is fed solidly into a large barrel and is pushed forward with a constant velocity by the screw. While passing through the barrel, the raw polymer is gradually heated until the fed polymer is melted. Finally, the melted material is pushed out of the machine to be processed (Su and Tsai, (1997)). In order to produce a high quality extrudates, the temperature along the barrel must be appropriately set and precisely controlled (Su and Tsai, (2001)).

In recent years, several temperature control techniques have been proposed. For instance, classical schemes such as PD and PID controllers and their adaptive variations (see Taur et. al, (1995), Petrovčič et al., (2013), Hamane et al., (2010)). However, it is wellknown that these controllers lack robustness towards time-varying disturbances. For this reason, non-linear control techniques have been developed (see for example, Yao et al., (2007), Yao et al., (2008), Peng and Wei, (2011a), Peng and Wei (2011b)). One of the most popular robust technique is the Sliding Mode Control (SMC). This method ensures finite time convergence and its robust towards parametric uncertainties and external disturbances as well. However, the main drawback of this technique is the undesirable chattering effect. In the study of $\mathrm{Su}$ and Tsai (2001), a discrete first order sliding mode controller for the temperature control is proposed.
To tune the developed algorithm, the authors propose an adaptive law. However, the adaption algorithm is complex and it has several controller gains to tune. Moreover, the method to tune the feedback controller gains is not explicitly given.

In this paper, based on the previous results of Su and Tsai (2001), an adaptive first order sliding mode control for regulation and trajectory tracking of temperature profiles is proposed. The adaptation law is based on the proposed technique by Plestan et al., (2010). In our paper, we modify the adaption scheme in order to develop a control law that does not need information about the system and is robust towards parametric uncertainties and external disturbances. To the best knowledge of the authors, this method has not been applied to control this type of systems yet. Moreover, compared to the work of $\mathrm{Su}$ and Tsai, (2001), we address the trajectory tracking problem. Finally, the proof of stability is proven by Lyapunov arguments.

The remainder of this paper is organized as follows: The temperature plastic extrusion mathematical description is reminded in Section 2. In Section 3, an adaptive first order sliding mode controller for regulation and trajectory tracking problem and its stability anlysis is presented. In order to demonstrate the effectiveness of the proposed control scheme, computer simulations are presented in Section 5. Finally, we make a brief conclusion on the paper in Section 6.

\section{Temperature Plastic Extrusion Mathematical Model}

The general energy balance equation in a stationary volume control defines that the rate of accumulation energy is equal to the rate of input and output energy difference. Thus, the energy balance equation, can be expressed as follows:

$\rho c_{p} \mathrm{~V}_{\mathrm{e}} \frac{\mathrm{dT}}{\mathrm{dt}}=\omega-\mathrm{hA}\left(\mathrm{T}-\mathrm{T}_{\infty}\right)$

where $T$ is the temperature, $T_{\infty}$ is the environment's temperature. $\rho$ and $c_{p}$ is the density and the specific heat of the material, respectively. $V_{e}$ is the filament output velocity, $A$ is the filament area, and $h$ is the heat transfer coefficient. 
Finally, $\omega$ is the input energy rate where $\omega>0$ is for heating and $\omega<0$ is for cooling. Then, arranging Eq. (1), yields to the following dynamic equation:

$\frac{\mathrm{dT}}{\mathrm{dt}}=\frac{h A}{\rho c_{p} V_{e}} \mathrm{~T}_{\infty}-\frac{h A}{\rho c_{p} V_{e}} \mathrm{~T}+\frac{\omega}{\rho c_{p} V_{e}}$

Note that the electric heaters and the coolers are considered as the thermal actuators of the term $\omega$. Considering that the actuator's internal dynamics is given by the following equation:

$\dot{\omega}=\gamma(\omega, \mathrm{u}, \mathrm{t})= \begin{cases}\gamma^{+}(\omega, u, t) & \text { if } u \geq 0 \\ \gamma^{-}(\omega, u, t) & \text { if } u<0\end{cases}$

Where the electric heaters are activated when the control signal $u>0$ and the cooling system is on when the term $u<0$. It is assumed that the functions $\gamma^{+}(\cdot)$ and $\gamma^{-}(\cdot)$ are nonlinear, unknown but bounded. Now, from Eq. (2), if we introduce the following variable:

$v=\frac{h A}{\rho V_{e} c_{p}} \mathrm{~T}_{\infty}+\frac{\omega}{\rho c_{p} V_{e}}$

Taking into account (4), the system (2) yields to the following single-channel dynamic system:

$\frac{d T}{d t}=-\frac{h A}{\rho c_{p} V_{e}} \mathrm{~T}+v$
$\frac{d v}{d t}=\frac{\gamma(\omega, u, t)}{\rho c_{p} V_{e}}+\xi_{0}(t)$

Where $\xi_{0}(t)$ is the unknown dynamics of $v$. Moreover, in order to improve the the given model, we introduce lumped disturbances in each channel of the proposed system. Thus, the system (5) can be expressed as follows:

$\frac{d T}{d t}=-\alpha_{1} \mathrm{~T}+v+\xi_{0}(t)$
$\frac{d v}{d t}=\alpha_{2} \gamma(\omega, u, t)+\xi_{2}(t)$

Where $\quad \alpha_{1}=h A / \rho c_{p} V_{e}$ and $\alpha_{2}=$ $1 / \rho c_{p} V_{e}$. Also, $\xi_{2}(t)$ contains the term $\xi_{0}(t)$ and any bounded external disturbance.
In real applications, it is well-known that the partial information about the system parameters is available only. Taking into account this issue, it is assumed that the system parameters $\alpha_{i}$ are partially known. Thus, the terms $\alpha_{i}$ are divided into two terms, i.e., $\alpha_{i}=$ $\hat{\alpha}_{i}-\tilde{\alpha}_{i}$, where $\hat{\alpha}_{i}$ is the nominal parameter and $\tilde{\alpha}_{i}$ represents the term that contains the unknown model parameter and model uncertainties, and it is defined as $\tilde{\alpha}_{i}=\hat{\alpha}_{i}-\alpha_{i}$. Finally, taking into account the given nomenclature, we can rewrite the system (6) as follows:

$$
\begin{aligned}
& \frac{d T}{d t}=-\hat{\alpha}_{1} \mathrm{~T}+v+\mathrm{d}_{1}(t) \\
& \frac{d v}{d t}=\hat{\alpha}_{2} \gamma(\omega, u, t)+\mathrm{d}_{2}(t)
\end{aligned}
$$

Where $\quad d_{i}(t) \forall i=1,2 \quad$ are the disturbance terms and contain the lumped external disturbances $\xi_{1}(t)$ and $\xi_{2}(t)$, and the parameter uncertainties. Moreover, this term is explicitly defined as

$d_{1}(t)=\xi_{1}(t)+\tilde{\alpha}_{1} T \quad$ and $\quad d_{2}(t)=\xi_{2}(t)-$ $\tilde{\alpha}_{2} \gamma(\cdot)$.

Remark 1. It is assumed that the disturbance term is bounded as follows:

$\left\|d_{i}(t)\right\| \leq \delta_{\mathrm{i}}$

Where $\delta_{i} \in \mathbb{R} \forall i=1,2$ is the positive upper bound of the disturbance but it is not known.

\section{Controller Design}

In this section, an adaptive controller based on first order sliding modes theory is designed. First, we introduce the sliding surface, $\sigma$, which depends on the error variable as follows:

$\sigma(t)=\dot{e}(\mathrm{t})+\lambda \mathrm{e}(\mathrm{t})$

Where $e(t)=T(t)-T^{d}(t)$ is the tracking error, $\dot{e}(t)$ is the time derivative of the error signal, $T(t)$ is the temperature and $T^{d}(t)$ is the desired temperature value. It is worth to note that $T^{d}(t)$ is a function in time because the proposed controller addresses the trajectory tracking problem. However, to solve the setpoint regulation, the desired value needs to be fixed to a constant value instead a function on time. 
Then, the proposed controller's objective is to drive the error value to zero, this means that $T(t) \rightarrow T^{d}(t)$ in finite time, in spite of the external disturbances and/or model uncertainties. This condition can be achieved by the following control law:

$u(t)=\frac{1}{\widehat{\alpha}_{2}}\left[\ddot{T}^{d}(t)+\hat{\alpha}_{1} \dot{T}(t)-\lambda \dot{e}-\widehat{K} \operatorname{sgn}(\sigma)\right]$

Where $\ddot{T}^{d}(t)$ is the second time derivative of $T(t), \operatorname{sgn}(\cdot)$ is the signum function and $\widehat{K}$ is the controller's adaptive gain, which its dynamics are given as follows:

$\dot{\hat{K}}= \begin{cases}\beta|\sigma|^{\frac{1}{2}} \operatorname{sgn}(|\sigma|-\mu) & \text { if } \widehat{K} \geq K_{\text {min }} \\ \kappa & \text { if } \widehat{K}<K_{\text {min }}\end{cases}$

Where $\beta, \mu, \kappa$ and $K_{\min }$ are positive constants. The stability of the proposed controller is given in the main theorem below.

Theorem 1. Let the temperature dynamics of the plastic extrusion process given by the Eq. (5). Taking into account the model uncertainties and the lumped external disturbance, the model (5) can be rewritten as the equivalent system (7). Suppose that the disturbance term $d_{i}(t)$ satisfies (8). Then for any initial condition $\sigma(0)$ the sliding surface $\sigma=0$ will be reached in finite time via the adaptive controller (10) with the adaptive gain selected as shown in Eq. (11).

Proof. Injecting the control law (10) into the time derivative of $\sigma$ given by Eq. (9), yields to:

$$
\dot{\sigma}=-\widehat{K} \operatorname{sgn}(|\sigma|-\mu)+\dot{d}_{1}(t)+d_{2}(t)
$$

Let us define the following candidate Lyapunov function as:

$$
V(\sigma, \widehat{K})=\frac{1}{2} \sigma^{2}+\frac{1}{2 \eta}\left(\widehat{K}-\widehat{K}^{*}\right)^{2}
$$

Where $\eta>0$. Also, note that the given function is positive and radially unbounded in almost everywhere except in the origin. Then, taking its time derivative, one can obtain the following expression:

$$
\begin{aligned}
& \dot{V}(\sigma, \widehat{K})=\sigma \dot{\sigma}+\frac{1}{\lambda}\left(\widehat{K}-\widehat{K}^{*}\right) \dot{\hat{K}}= \\
& \sigma\left[-\widehat{K} \operatorname{sgn}(|\sigma|-\mu)+\dot{d}_{1}(t)+d_{2}(t)\right]+ \\
& +\frac{1}{\eta}\left(\widehat{K}-\widehat{K}^{*}\right)\left(\beta|\sigma|^{\frac{1}{2}} \operatorname{sgn}(|\sigma|-\mu)\right) \\
& \leq-\widehat{K}|\sigma|-\delta_{3}|\sigma|+\widehat{K}^{*}|\sigma|-\widehat{K}^{*}|\sigma| \\
& +\frac{1}{2}\left(\widehat{K}-\widehat{K}^{*}\right)\left(\beta \operatorname{sgn}|\sigma|^{\frac{1}{2}}(|\sigma|-\mu)\right)
\end{aligned}
$$

Remark 2. Note that the boundary of the disturbances exists, but is unknown. This is:

$$
\left|\dot{d}_{1}(t)+d_{2}(t)\right| \leq \delta_{1}+\delta_{2}=\delta_{3}
$$

Considering that there exist a $K^{*}>0$ such that $\widehat{K}-K^{*}<0$ for all $t \geq 0$, then, it is possible to rewrite the Eq. (14) as follows:

$$
\begin{aligned}
& \dot{V}(\cdot) \leq\left(\delta_{3}-\widehat{K}^{*}\right)|\sigma|+\varsigma\left|\widehat{K}-\widehat{K}^{*}\right|-\varsigma\left|\widehat{K}-\widehat{K}^{*}\right| \\
& +\left(\widehat{K}-\widehat{K}^{*}\right)\left(-|\sigma|+\frac{\beta}{\eta} \operatorname{sgn}|\sigma|^{\frac{1}{2}}(|\sigma|-\mu)\right) \\
& =-\left(\widehat{K}^{*}-\delta_{3}\right)|\sigma|-\varsigma\left|\widehat{K}-\widehat{K}^{*}\right|- \\
& -\left|\widehat{K}-\widehat{K}^{*}\right|\left(-|\sigma|+\frac{\beta}{\eta} \operatorname{sgn}|\sigma|^{\frac{1}{2}}(|\sigma|-\mu)-\varsigma\right)
\end{aligned}
$$

Let us define $\pi_{1}=\widehat{K}^{*}-\delta_{3}$ and $\pi_{2}=$ $\left|\widehat{K}-\widehat{K}^{*}\right|\left(-|\sigma|+\frac{\beta}{\eta} \operatorname{sgn}|\sigma|^{\frac{1}{2}}(|\sigma|-\mu)-\varphi\right)$.

Then, the above equation can be rewritten as:

$$
\begin{aligned}
& \dot{V}(\sigma, \widehat{K})=-\pi_{1}|\sigma|-\varsigma\left|\widehat{K}-\widehat{K}^{*}\right|-\pi_{2} \\
& \dot{V}(\sigma, \widehat{K})=-\pi_{1} \sqrt{2} \frac{|\sigma|}{\sqrt{2}}-\varsigma \sqrt{2 \eta} \frac{\left|\widehat{K}-\widehat{K}^{*}\right|}{\sqrt{2 \eta}}-\pi_{2} \\
& \dot{V}(\cdot) \leq-\min \left\{\pi_{1} \sqrt{2}, \varsigma \sqrt{2 \eta}\right\}\left(\frac{|\sigma|}{\sqrt{2}}+\frac{\left|\widehat{K}-\widehat{K}^{*}\right|}{\sqrt{2 \eta}}\right)-\pi_{2} \\
& \dot{V}(\sigma, \widehat{K}) \leq-\chi \mathrm{V}^{\frac{1}{2}}-\pi_{2}
\end{aligned}
$$

Where $\quad \chi=\min \left\{\pi_{2} \sqrt{2}, \varsigma \sqrt{2} \eta\right\} . \quad$ The analysis of the behavior of the time derivative of $V$ can be addressed by considering two cases only. On the one hand, if we consider that $\sigma>$ $\mu$, this means that $\pi_{2}$ is:

$$
\pi_{2}=-\mu+\frac{\beta}{\eta} \mu^{\frac{1}{2}}-\varsigma>0
$$

From Eq. (18), it is easy to see that the following condition holds:

$\eta=\frac{\beta \mu^{\frac{1}{2}}}{\mu+\varsigma}$

This condition ensures the negativeness of the function $\dot{V}=(\sigma, \widehat{K})$ when the trajectories enter in the region $\widehat{K}>K_{\min }$. 
On the other hand, when $|\sigma|<\mu$, it is not possible to prove the negativeness of the function $\dot{V}$. However, based on the structure of the dynamics of $\dot{\hat{K}}$, when the system trajectories are in this region, the gain will increase as time grows up and, eventually, the value of $\sigma$ will satisfy $|\sigma|>\mu$ and the function $\dot{V}(x)$ will be negative definite. Finally, the sliding surface $\sigma=0$ will be reached in finite time in spite of parametric uncertainties and bounded external disturbances. This means that the $T(t)$ will tend to the desired value $T^{d}(t)$ as time increases.

Remark 3. The main theorem given above ensures the finite time convergence of the system trajectories to the reference signal in spite of parametric uncertainties and external disturbances. However, it is well-known that the main drawback of the first order sliding mode control is the chattering effect. The chattering amplitude is related to the controller gain, this means that if the gain is set to a high value, the amplitude of the chattering effect will be large. Nevertheless, we can consider different approaches in order to reduce the chattering into the control signal. For instance, a sigmoid profile is used instead the signum function, or a Low Pass Filter (LPF) is designed to alleviate the chattering effect. In this work, we design the LPF as follows:

$\mathrm{Q} \dot{v}(\mathrm{t})+v(\mathrm{t})=\mathrm{u}(\mathrm{t})$

Where $\varrho$ is a small positive constant. Note that (20) represents the filter dynamics and the variable $v$ will be the new control signal.

\section{Simulation Results}

In this section, the robustness of the proposed controller is demonstrated through computer simulations. The simulations were performed in MATLAB software. The parameters used in the simulations are synthesized in Table I. Also, we consider the temperature profile for the PET material. Finally, the controller parameters are synthesized in Table II.

\begin{tabular}{|l|l|}
\hline Parameter & \multicolumn{1}{c|}{ Value } \\
\hline$\rho$ & $1380\left[\mathrm{Kg} / \mathrm{m}^{3}\right]$ \\
\hline$c_{p}$ & $1350[\mathrm{~kJ} /(\mathrm{kg} * \mathrm{~K})]$ \\
\hline$V_{e}$ & $0.0207[\mathrm{~m} / \mathrm{s}]$ \\
\hline$h$ & $133\left[\mathrm{~W} / \mathrm{m}^{2} \mathrm{~K}\right]$ \\
\hline$T_{\infty}$ & $25\left[{ }^{\circ} \mathrm{C}\right]$ \\
\hline
\end{tabular}

Table 1 System Parameters when PET material is considered

Source: own elaboration

\begin{tabular}{|l|r|}
\hline Parameter & Value \\
\hline$\lambda$ & 1 \\
\hline$\beta$ & 0.005 \\
\hline$\mu$ & 1 \\
\hline$\kappa$ & 0.1 \\
\hline$K_{\min }$ & 0.01 \\
\hline$\varrho$ & 2 \\
\hline
\end{tabular}

Table 2 Control Parameter used in the simulation. Source: own elaboration

In this study, we consider the simulation for the temperature dynamics into the plastic extruder feed zone only. Note that the proposed methodology can be applied to each heat zone of the extruder. In fact, the robustness of the controller reduces the coupling effect when several controllers are considered.

In order to demonstrate the positive properties of the proposed controller, four scenarios arise as:

Nominal Scenario: In this case, the setpoint regulation is addressed without considering external disturbances or parametric uncertainties.

- Controller's robustness towards parametric uncertainties: In this case, it is assumed that the model parameters are unknown, and therefore they are not considered into the controller design.

- Controller's robustness towards external disturbances: In this scenario, it is considered external disturbances such as environmental temperature changes, sudden changes in the system parameters, actuation failure and so on.

Trajectory tracking test: The reference signal is a time varying function. In this test, external disturbances and model uncertainties are considered. 


\section{Nominal Case}

In this scenario, the full knowledge of the system parameters is considered. Moreover, we do not take into account the lumped external disturbance, i.e., $d_{i}(t)=0$. The reference signal is fixed to $T^{d}(t)=260{ }^{\circ} \mathrm{C}$. Finally, the control law tested is given by Eq. (10).

The set-point regulation results are shown in Fig. 1. From this Figure, we can observe that the temperature profile goes to the reference signal in almost 2500 seconds, which is a reasonable amount of time compared to other studies (see Su and Tsai, (2001)). The error signal plot is shown in the middle of Fig. 1. From this Figure, it can be observed that the tracking error vanishes as time increases. At the right lower part of Fig. 1, we can observe the controller's gain adaption. Finally, the left lower part of Fig. 1 shows the controller's signal evolution. It is possible to observe that the amplitude of the chattering effect was reduced using the LPF.

\section{Controller Robustness Towards Parametric Uncertainties}

In this scenario, the model parameters are unknown. For this reason, we modify the control law (10) as follows:

$\mathrm{u}(\mathrm{t})=\frac{1}{\widehat{\alpha}_{2}}[-\lambda \dot{e}-\widehat{K} \operatorname{sgn}(\sigma)]$

Note that we have neglected the term $\hat{f}(x)=\ddot{T}^{d}(t)+\hat{\alpha}_{1} \dot{T}(t)$, which is related to the system's parameters.

Figure 2 shows the controller performance when parameter uncertainties are considered. As we can see, the trajectory system tends to the reference value even in presence of model uncertainties. In fact, the behavior is similar to the previous case, and the system converge to the $T^{d}$ in almost 2500 seconds. The error plot is shown in the middle of Fig. 2. The evolution of the control signal is displayed in the left lower part of Fig. 2. As expected, the chattering amplitude is increased.

\section{Controller's Robustness Towards External} Disturbances
In this case, external disturbances are considered. We consider both cases: the constant disturbance, i.e., $d_{1}(t)=10$ and a time varying disturbance such as $d_{1}(t)=$ $5 \sin (2 \pi f t)$ where the period was set to $T_{f}=$ 50 seconds, and $f=1 / T_{f}$ is the frequency signal. The disturbance was introduced from 2500 to 4000 seconds.

The performance of the controller towards the constant disturbance can be seen in Figure 3. In the middle of Fig. 3 shows the convergence of the error to the origin. The evolution of the controller signal is displayed at the bottom of Fig. 3. Finally, the gain evolution is shown in the left lower part of the Fig. 3.

Fig. 4 shows the controller's response when time-varying external disturbance is considered. In the upper part of Fig. 4, we can see that the trajectory system reaches the reference in spite of the external disturbance at a time of 2500 seconds. In this plot, it is possible to observe that the disturbance is not completely suppressed. However, the controller's response is enough to minimize the negative impact of the disturbance. In the middle of Fig. 4, the error plot is depicted. The evolution of the controller signal is displayed in the left lower part of Fig. 4. From this Figure, we can observe that the chattering amplitude is increased because the controller needs to adjust its gain to counteract the disturbance effect, when the gain is increased, the chattering amplitude grows up as well.

\section{Trajectory Tracking test}

In this scenario, the time-varying profile trajectory tracking is proposed. The desired profile is defined as $T^{d}(t)=260+$ $5 \cos (2 \pi f t)$ where $f$ is the signal frequency with a period of $T_{f}=100$ seconds. The reference signal is introduced into the system dynamics from 500 seconds to the end of the simulation. In this case, we have assumed that the temperature of the extrusion process change as different material is introduced into the extrusion machine. 
The trajectory tracking task is shown at the top of Fig. 5, as we can see, the controller converges to the reference in finite time. Also, in the middle of Fig. 5, the error plot is displayed. Finally, the control signal is shown at the bottom of Fig. 5. It can be observed that the controller's effort is large when the desired reference changes. In the right bottom side, we can observe the evolution of the controller's feedback gain.

\section{Conclusion}

In this paper, an adaptive first order sliding mode control has been proposed. The adaptive controller is designed to address the trajectory tracking problem but it can be used for the setpoint regulation paradigm. The stability analysis for the resulting closed-loop system for trajectory tracking has been addressed. The proposed controller has been tested by computer simulations in MATLAB for several scenarios to prove its robustness towards disturbances. The simulation experiments results demonstrate the effectiveness, and robustness of the proposed scheme to uncertainties on the parameters of the system and to external disturbances, as well. In this manuscript, constant disturbances and timevarying external disturbances were considered. The design of the discrete adaptive SMC is a part of future work.

\section{Annexes}
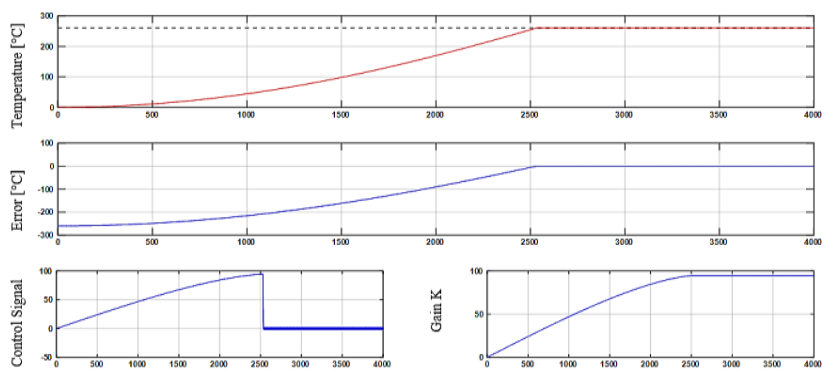

Figure 1 Nominal Case

Source: own work [MatLab]

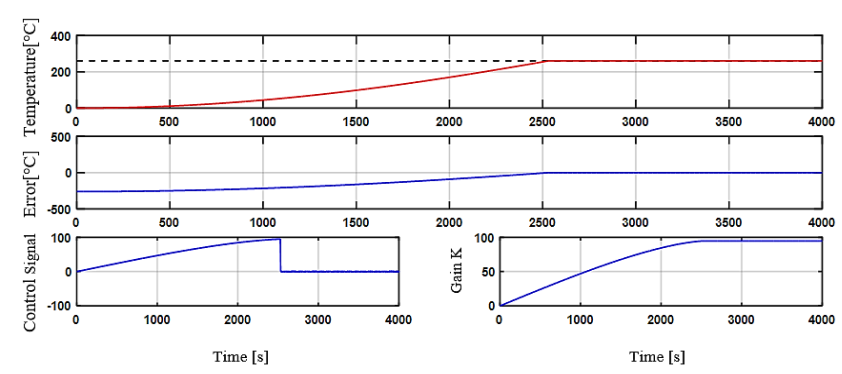

Figure 2 Controller robustness towards parametric uncertainties

Source: own work [MatLab]

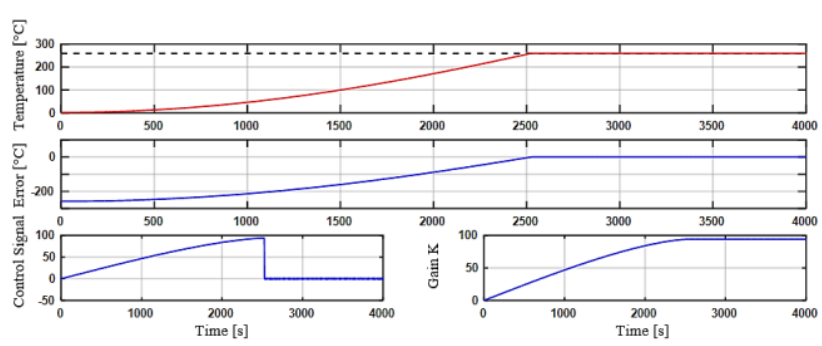

Figure 3 Controller robustness towards constant disturbance

Source: own work [MatLab]

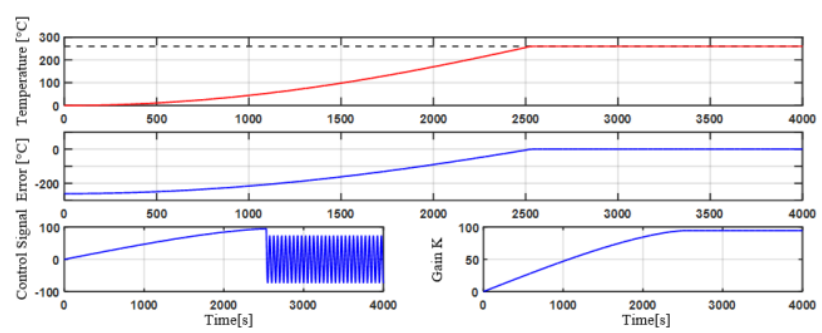

Figure 4 Controller robustness towards time-varying disturbance

Source: own work [MatLab]

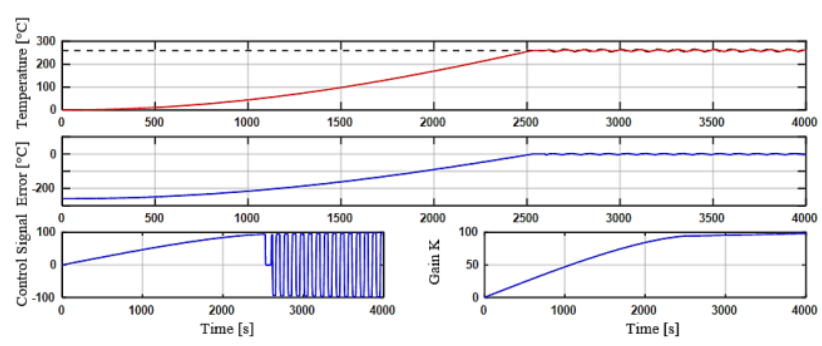

Figure 5 Trajectory tracking test Source: own work [MatLab]

\section{References}

Guerrero Moro, C., Escalona Mendoza, R. A., \& Tapia Hernández, G. (2020). Diseño e instalación de sistema de riego automatizado para orquídea oncidium sphacelatum en invernadero de la Universidad Autónoma de Chapingo.

Guzmán Orrala, J. K. (2020). Implementación de un transportador de producto terminado con un sistema automatizado de conteo y empaquetado con filtro de comprobación por balanza empleando el uso de un microcontrolador.

Hamane, H., Matuki, K., Hiroki, F., \& Miyazaki, K. (2010). Thermal MIMO controller for setpoint regulation and load disturbance rejection. Control Engineering Practice, 18(2), 198-208.

GUERRERO, Jesús, GONZÁLEZ, Julio and CHIMAL, Martin. Adaptive temperature controller for plastic extrusion process. Journal of Technological Prototypes. 2020 
Hammond Fernández, T. (2020). Sistema de aspersión de neumáticos para la circulación del automóvil en pavimentos nevados.

Marquina Castro, J. E. (2020). Efecto de la instrumentación mecanizada con el uso de una lima de acabado en la magnitud de gutapercha residual a nivel apical del conducto radicular de premolares en proceso de retratamiento.

Peng, Y., \& Wei, W. (2011). Melt temperature dynamic control strategy of injection molding machine based on variable structure control and iterative learning control. Journal of Polymer Engineering, 31(6-7), 473-478.

Peng, Y., \& Wei, W. (2011). Melt temperature learning control of injection molding process based on CMAC neural network. Journal of Polymer Engineering, 31(1), 45-52.

Petrovčič, J., \& Vrančić, D. (2013). Temperature Control in a Plastic Extruder Control System. In Case Studies in Control (pp. 157-183). Springer, London.

Plestan, F., Shtessel, Y., Bregeault, V., \& Poznyak, A. (2010). New methodologies for adaptive sliding mode control. International journal of control, 83(9), 1907-1919

Su, W. C., \& Tsai, C. C. (1997, June). Discretetime VSS temperature control for a plastic extrusion process with water cooling systems. In Proceedings of the 1997 American Control Conference (Cat. No. 97CH36041) (Vol. 4, pp. 2571-2575). IEEE.

Su, W. C., \& Tsai, C. C. (2001). Discrete-time VSS temperature control for a plastic extrusion process with water cooling systems. IEEE Transactions on Control Systems Technology, 9(4), 618-623.

Taur, J. S., Tao, C. W., \& Tsai, C. C. (1995, May). Temperature control of a plastic extrusion barrel using PID fuzzy controllers. In Proceedings IEEE Conference on Industrial Automation and Control Emerging Technology Applications (pp. 370-375). IEEE.
Urra-Sanhueza, C. (2020). Recovery and stabilization of phenolic antioxidants and their potential bioactivity as antimicrobial and antiproliferative agents, from residues of the avocado agroindustry (Persea americana, Hass variety).

Yao, K., \& Gao, F. (2007). Optimal start-up control of injection molding barrel temperature.

Yao, K., Gao, F., \& Allgöwer, F. (2008). Barrel temperature control during operation transition in injection molding. Control Engineering Practice, 16(11), 1259-1264. Polymer Engineering \& Science, 47(3), 254-261. 